\title{
High glucose treatment promotes extracellular matrix proteome remodeling in Müller glial cells
}

\author{
Sandra Sagmeister $^{1,2}$, Juliane Merl-Pham ${ }^{2}$, Agnese Petrera ${ }^{2}$, Cornelia A Deeg ${ }^{\text {Corresp., } 1}$, Stefanie M Hauck ${ }^{\text {Corresp. } 2}$ \\ ${ }^{1}$ Chair of Physiology, Department of Veterinary Sciences, LMU Munich, Martinsried, Germany \\ 2 Research Unit Protein Science and Metabolomics and Proteomics Core Facility, Helmholtz Center Munich, German Research Center for Environmental \\ Health $\mathrm{GmbH}$, Munich, Germany \\ Corresponding Authors: Cornelia A Deeg, Stefanie M Hauck \\ Email address: Cornelia.Deeg@Imu.de, hauck@helmholtz-muenchen.de
}

Background. The underlying pathomechanisms in diabetic retinopathy (DR) remain incompletely understood. The aim of this study was to add to the current knowledge about the particular role of retinal Müller glial cells (RMG) in the initial processes of DR.

Methods. Applying a quantitative proteomic workflow, we investigated changes of primary porcine RMG under short term high glucose treatment as well as glycolysis inhibition treatment. Results. We revealed significant changes in RMG proteome primarily in proteins building the extracellular matrix (ECM) indicating fundamental remodeling processes of ECM as novel rapid response to high glucose treatment. Among others, Osteopontin (SPP1) as well as its interacting integrins were significantly downregulated and organotypic retinal explant culture confirmed the selective loss of SPP1 in RMG upon treatment. Since SPP1 in the retina has been described neuroprotective for photoreceptors and functions against experimentally induced cell swelling, it's rapid loss under diabetic conditions may point to a direct involvement of RMG to the early neurodegenerative processes driving DR. Data are available via ProteomeXchange with identifier PXD015879. 


\section{High glucose treatment promotes extracellular matrix}

\section{2 proteome remodeling in Müller glial cells}

3

4 Sandra Sagmeister ${ }^{1,2}$, Juliane Merl-Pham ${ }^{1}$, Agnese Petrera ${ }^{1}$, Cornelia A Deeg²*, Stefanie M

5 Hauck $^{1 *}$

6

$7 \quad{ }^{1}$ Research Unit Protein Science and Metabolomics and Proteomics Core Facility, Helmholtz

8 Center Munich, German Research Center for Environmental Health GmbH, Munich, Germany

$9 \quad{ }^{2}$ Chair of Physiology, Department of Veterinary Sciences, LMU Munich, Martinsried, Germany

11 Corresponding Author:

12 Stefanie M Hauck

13 Email address: hauck@helmholtz-muenchen.de

14 Cornelia A. Deeg

15 Email address: deeg@tiph.vetmed.uni-muenchen.de

\section{Abstract}

Background. The underlying pathomechanisms in diabetic retinopathy (DR) remain incompletely understood. The aim of this study was to add to the current knowledge about the particular role of retinal Müller glial cells (RMG) in the initial processes of DR.

Methods. Applying a quantitative proteomic workflow, we investigated changes of primary porcine RMG under short term high glucose treatment as well as glycolysis inhibition treatment. Results. We revealed significant changes in RMG proteome primarily in proteins building the extracellular matrix (ECM) indicating fundamental remodeling processes of ECM as novel rapid response to high glucose treatment. Among others, Osteopontin (SPP1) as well as its interacting integrins were significantly downregulated and organotypic retinal explant culture confirmed the selective loss of SPP1 in RMG upon treatment. Since SPP1 in the retina has been described neuroprotective for photoreceptors and functions against experimentally induced cell swelling, it's rapid loss under diabetic conditions may point to a direct involvement of RMG to the early 
31 neurodegenerative processes driving DR. Data are available via ProteomeXchange with 32 identifier PXD015879.

33

\section{Introduction}

The worldwide prevalence for diabetes in adults is increasing and is predicted to exceed ten percent of world population by 2040 (Ogurtsova et al. 2017). Diabetic retinopathy (DR) is a severe and frequent comorbidity of diabetes and a leading cause of vision loss (Ducos et al. 2020; Graue-Hernandez et al. 2020; Leasher et al. 2016). However, the underlying pathomechanisms remain to be comprehensively elucidated (Mesquida et al. 2019). DR was long proposed to be mainly a microvascular disease, but is today perceived rather as a neurodegenerative disorder in its early stages (Kadlubowska et al. 2016). Neurodegeneration occurs even before any vascular alterations can be detected and cannot be reversed (Kadlubowska et al. 2016). Thus, there is an urgent need to learn about initial pathomechanisms involved in the early neurodegenerative process. Retinal Müller glial cells (RMG) are a major source for neuroprotective signals (Del Rio et al. 2011; Hauck et al. 2008; Hauck et al. 2014) also in the diabetic mouse retina (Fu et al. 2015). Thus, the novel concept of modulating RMG to improve or re-establish retinal function is gaining increasing attention. A detailed understanding of RMG participation in the onset of neurodegeneration could guide novel therapeutic intervention strategies and, in best case, help prevent neurodegeneration.

The retina is composed of a complex network of highly specialized cells (Mazade \& Eggers 2020). RMG are the dominant macroglial cell type within the retina and retinal health depends on their undisturbed functions (Bringmann et al. 2006). RMG seem to be fundamentally involved in pathologic mechanism of DR (Coughlin et al. 2017). They mediate the local inflammatory response, trigger pathological neovascularization and fibrosis, participate in damage of blood retinal barrier and downregulate potassium channels vital to retinal fluid homeostasis and synaptic function (Coughlin et al. 2017; Sorrentino et al. 2016; Subirada et al. 2018). However, little is known about the exact mechanism in RMG and changes on protein level have not yet been studied in DR (McDowell et al. 2018).

Due to the high anatomical and functional similarities of pig and human eyes, pig ocular tissue has proven a promising model for translational research (Giese et al. 2020; Kleinwort et al. 2017; Menduni et al. 2018; Renner et al. 2020). While other animal models fall short in mimicking the 
62 clinical background of DR in man (Mi et al. 2014), the diabetic INSC94Y transgenic pig is the first

63 model to represent a broad variety of the human retinal pathology (Kleinwort et al. 2017;

64 Weigand et al. 2020). We hence established a cell culture model for DR on isolated porcine

65 RMG by applying diabetic conditions in vitro. Hyperglycemia is considered as major trigger of

66 pathological events in DR (Mathebula 2018) and streptozotocin-induced diabetes is known to

67 increase retinal glucose levels in diabetic rats (Ola et al. 2006; Zhang et al. 2003). We therefore

68 applied short term high glucose treatment to simulate early hyperglycemic conditions in diabetes.

69 To meet another form of disturbed glucose metabolism, we also applied 2-deoxyglucose

70 treatment to simulate glycolysis inhibition (Valdes et al. 2016). There are indications that

71 decreased glycolysis or inactivity of glycolytic enzymes are another critical matter in the

72 development of DR in diabetic rats (Kanwar \& Kowluru 2009; Ola et al. 2006; Thomas et al.

73 2019).

74 In this study, we explored proteomic changes of porcine RMG in a diabetic cell culture model

75 and contribute to understanding RMG mediated pathomechanisms in DR. Studying RMG

76 responses to short term high glucose and glycolysis inhibition treatment on protein level allowed

77 us to assess initial involvement of RMG in early stages of DR. The proteomic data was

78 confirmed by immunohistochemical staining on porcine retinal organotypic explant cultures.

79

80 Materials \& Methods

81 Preparation of primary RMG

82 No experimental animals were used in this study, eyes from healthy adult pigs were received

83 fresh from a local abattoir. The eyes were removed from the animals within five minutes after

84 death and kept on ice in $\mathrm{CO}_{2}$-independent medium (Thermo Fisher Scientific, Ulm, Germany)

85 until preparation started within one hour. Collection and use of porcine eyes from the abattoir

86 was approved for purposes of scientific research by the appropriate board of the veterinary

87 inspection office Munich, Germany (permit number: DE 09162 0008-21). Eight neuroretinae

88 from eight animals were prepared and primary RMG were isolated as previously described

89 (Hauck, et al., 2003). Briefly, major blood vessels were removed, each retina was mechanically

90 cut and retinal pieces were washed twice in Ringer's solution (Millipore Sigma, Darmstadt,

91 Germany). Dissociation was obtained by treating each retina with $2.2 \mathrm{U}$ of activated papain 
92 (Worthington Biochemical, Troisdorf, Germany) for 12 minutes at $37^{\circ} \mathrm{C}$. Papain enzyme activity 93 was stopped by the addition of DMEM Glutamax (Thermo Fisher Scientific) with 10\% fetal calf 94 serum (Thermo Fisher Scientific). Then, 160 Kunitz-units of DNase (Millipore Sigma,

95 Darmstadt, Germany) was added and the tissue was further dissociated by gentle trituration using 96 a fire-polished Pasteur pipette. Dissociated cells were pooled, resuspended and plated in six well 97 format (cells from about two retinae per six well plate) in DMEM Glutamax containing $5.6 \mathrm{mM}$ 98 glucose in addition of 10\% fetal calf serum and 1\% Penicillin-Streptomycin (Thermo Fisher 99 Scientific). After 24 hours at $37^{\circ} \mathrm{C}$ in $5 \% \mathrm{CO}_{2}$, medium was exchanged and debris, nonattached 100 or loosely attached cells were removed by agitation (panning). Cells were cultivated for two 101 weeks without passaging and DMEM Glutamax culture medium was exchanged every 48 to 72 102 hours. Under these treatment conditions, porcine RMG cells are rapidly dominating the culture 103 and reach purity and 80\% confluence after two weeks in culture (Hauck et al. 2003).

104 The time point after which confluence is reached strongly depends on the total number of seeded 105 cells. We spread the cells derived from two porcine eyes onto one 6well culture dish and 106 reproducibly reach $80 \%$ confluence after 14 days (100\% confluence is never reached in these 107 cultures). We chose this time point for our studies because this was the earliest consistent time 108 point with reasonably high cell yields for proteomic workflows. However, growth of RMG in 109 vitro goes along with trans-differentiation during culturing concomitant with strong

110 downregulation of prototype RMG marker proteins, like glutaminsynthetase (GS) (Hauck et al., 111 2003). The remaining expression of GS after only 7 days in culture (Hauck et al., 2003) is mainly 112 due to remaining resting cells which have not yet started growing (and simultaneously loosing 113 RMG markers). Since the mixture of non-growing and growing, yet de-differentiated cells is a 114 rather unstable experimental condition, we chose the time point 14 days, where the cells appear 115 homogeneously fibroblast-like, even though they have undergone trans-differentiation.

116 Cells were imaged by differential interference contrast on a Leica DMi8 microscope with the HC 117 PL Fluotar L 20x/0.40 DRY objective lens using a Leica DFC365FX-722433014 camera and 118 processed by the Leica Application Suite LASX (version 3.03, Leica, Wetzlar, Germany) (Fig1).

119 Diabetic treatment of RMG

120 Experiments were performed after 14 days in culture when cells reached a confluence of 121 approximately 80\%. Diabetic treatment was performed for 72 hours, with replacement of the 122 respective culture medium every 24 hours. The cells under normoglycemic conditions $(\mathrm{n}=6$ 
123 replicates, represented by different culture wells) were incubated at physiologic glucose level of

$1245.6 \mathrm{mM}$ resembling the physiologic blood glucose level in human and porcine species (Renner,

125 et al., 2013). The cells under high glucose treatment conditions aiming to simulate

126 hyperglycemic conditions ( $\mathrm{n}=6$ replicates) were cultured with $35 \mathrm{mM}$ glucose representing a

127 severe hyperglycemic state in diabetic patients (Yu et al. 2008; Zhu et al. 2015) by addition of

128 sterile filtered D-glucose (Millipore Sigma, Darmstadt, Germany). The cells under glycolysis

129 inhibition ( $\mathrm{n}=6$ replicates) were cultured under normoglycemic conditions with addition of 20

130 mM 2-deoxy-D-glucose (2-DG, Millipore Sigma, Darmstadt, Germany) during the last 24 of the

13172 hours. 2-DG is a synthetic glucose analog that is assimilated and subsequently phosphorylated

132 by mammalian RMG in vitro (Poitry-Yamate \& Tsacopoulos 1991; Toft-Kehler et al. 2017).

133 Uptake of 2-DG in mammalian (cancer) cells competes with glucose, as it is transferred by

134 glucose transporters (GLUTs) (Zhang et al. 2016). Phosphorylated 2-DG cannot be further

135 metabolized and accumulates leading to non-competitive inhibition of hexokinase, thus

136 disrupting glycolysis. In mouse retinae, a concentration of $1 \mathrm{mM}$ 2-DG in the absence of glucose

137 is sufficient to almost completely disrupt energy production ex vivo within 45 minutes (Chertov

138 et al. 2011). In the presence of glucose, 2-DG was applied in a ratio of 1:1 competing glucose to

139 achieve glycolysis inhibition within 24 hours (Valdes et al. 2016). In this experiment we applied

140 an about 3.5 times excess of 2-DG compared to the glucose level to achieve glycolysis inhibition

141 (20 mM 2-DG and $5.6 \mathrm{mM}$ glucose). Wells representing the different treatments were

142 randomized across three six well plates. Viability of cells at the end of the experiment was

143 examined under microscope and estimated over $80 \%$.

144

145 Sample preparation for mass spectrometry

146 After treatment, cells were washed trice in ice cold phosphate buffered saline (PBS). Plates were 147 put on ice and $100 \mu l$ lysis buffer (PBS with 1\% NP40, Roche Diagnostics, Mannheim,

148 Germany) was added per well. After two minutes of incubation, cells from each well were

149 scraped and collected separately. Lysis was performed by shear force and ultrasonication

150 followed by centrifugation for ten minutes at $10.000 \mathrm{rcf}$. Protein concentration was determined

151 by Bradford assay and equal total protein amounts $(10 \mu \mathrm{g})$ per replicate were digested with a

152 modified FASP procedure (Grosche et al. 2016; Wisniewski et al. 2009). Briefly, protein lysates

153 were diluted with ammonium bicarbonate buffer (Millipore Sigma, Darmstadt, Germany) to a

154 final volume of $400 \mu 1$, followed by reduction using $1 \mu 1$ of $1 \mathrm{M}$ dithiothreitol (Millipore Sigma,

155 Darmstadt, Germany) for $30 \mathrm{~min}$ at $60^{\circ} \mathrm{C}$. After cooling down to room temperature, $8 \mathrm{M}$ urea 
156 buffer $\mathrm{pH} 8.5$ (Millipore Sigma, Massachusetts, USA) was added to a final volume of $1 \mathrm{ml}$ and 157 proteins were carbamidomethlylated with $10 \mu \mathrm{l}$ of $300 \mathrm{mM}$ iodoacetamide (Millipore Sigma, 158 Darmstadt, Germany) for 30 minutes at room temperature in the dark. Two $\mu 1$ of $1 \mathrm{M}$

159

160 161

162 163 164 165 166 167 168 dithiothreitol was added to quench unreacted iodoacetamide, and protein lysates passed $30 \mathrm{kDa}$ centrifugal filters, Vivacon 500 (Sartorius, Göttingen, Germany). After washing three times with $200 \mu 1$ of urea buffer and three times with $100 \mu 1$ of $50 \mathrm{mM}$ ammonium bicarbonate buffer, the proteins on the filters were subjected to a two hours digest at room temperature with $0.5 \mathrm{~g}$ of Lysyl Endopeptidase (Wako, Osaka, Japan) followed by tryptic digest (1g of trypsin, Promega, Madison, USA) over-night at $37^{\circ} \mathrm{C}$. Peptides were collected by centrifugation through the filter and acidified with trifluoroacetic acid (Applied Biosystems, Foster City, USA) to a final pH 2.

\section{LC-MS/MS Analysis}

Approximately $0.5 \mu \mathrm{g}$ of peptides per sample were measured in a randomized fashion on a QExactive HF mass spectrometer online coupled to an Ultimate 3000 nano-RSLC (Thermo Fisher Scientific, Ulm, Germany) in data-independent acquisition (DIA) mode as previously described (Lepper et al. 2018; Mattugini et al. 2018). Briefly, peptides were loaded automatically on a trap column $(300 \mu \mathrm{m}$ inner diameter $\times 5$ mm, Acclaim PepMap100 C18, $5 \mu \mathrm{m}, 100 \AA \AA$; LC Packings,

Sunnyvale, USA) prior to C18 reversed phase chromatography on the analytical column

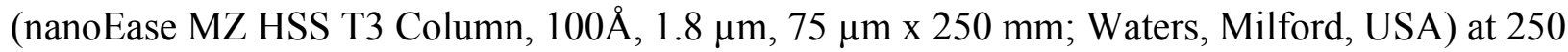
$\mathrm{nl} / \mathrm{min}$ flow rate in a 105 minute non-linear acetonitrile gradient from 3 to $40 \%$ in $0.1 \%$ formic acid. Profile precursor spectra from 300 to $1650 \mathrm{~m} / \mathrm{z}$ were recorded at 120,000 resolution and a maximum injection time of $120 \mathrm{~ms}$ for achieving an automatic gain control (AGC) value of $3 \mathrm{e} 6$. Subsequently fragment spectra were recorded in 37 overlapping DIA isolation windows (1 Da overlap on each side) of variable size covering in total 300 to $1650 \mathrm{~m} / \mathrm{z}$, each at 30,000 resolution with an AGC target of $3 \mathrm{e} 6$ and a normalized collision energy of 27.

\section{Quantitative Analysis}

The recorded raw files were analyzed using the Spectronaut software (version 12, Biognosys, Schlieren, Switzerland (Bruderer, et al., 2015, Bruderer, et al., 2016) with a peptide identification false discovery rate setting of $<1 \%$, using an in-house pig spectral library which was generated using Biognosys Spectronaut 12 and the Ensembl Pig database (release 75, Sscrofa10.2). Quantification was based on MS2 area levels of all unique proteotypic peptides per protein fulfilling the percentile 0.2 setting. Normalized protein quantifications were exported and used 
187 for calculations of fold-changes and significance values. Each group originally included six

188 replicates; in the normoglycemic control group two replicates were excluded due to a deviating 189 abundance response that could not be solved by normalization.

190 Statistics, Graphics, Principal component analysis, Pathway enrichment analysis, Volcano 191 plots, Venn diagram and Network generation

192 To analyze the differential protein abundance between the diabetic treatment groups and the 193 control group, a two-tailed, unpaired student's t-test was performed on log2 transformed 194 normalized abundances. Proteins were considered significantly changed upon the following 195 criteria: (a) quantified with $\geq 2$ unique peptides and (b) t-test $p$-value $<0.05$. Additionally, a fold 196 change cut-off of $\geq 1.33$-fold or $\leq 0.75$-fold was applied. To justify the chosen fold change cut197 off, we calculated the average variation coefficient CV of the individual groups (resulting in 198 average CVs of $13.2 \%$ in the normoglycemic, $12.0 \%$ in the high glucose and $14.1 \%$ in the 199 glycolysis inhibition group) and chose the fold change cut-off to exceed twice the maximal group $200 \mathrm{CV}$.

201 The workflow (Fig1) was designed using the medical image bank Servier Medical Art, which is 202 available under the Creative Commonslicense CC-BY (https://smart.servier.com/).

203 Pathway enrichment analysis (Fig2) was performed separately for the hyperglycemic and the 204 glycolysis inhibition treatment group with Genomatix Generanker (Genomatix Software GmbH, 205 Munich, Germany, http://www.genomatix.de) using EIDorado (version 04-2019) and Literature 206 Mining Database (version 02-2019). Inclusion criteria for proteins were (a) p-value $<0.05$, (b) 207 quantification with $\geq 2$ unique peptides and (c) $\mathrm{a} \geq 1.33$ fold or $\leq 0.75$-fold differential 208 abundance level. Gene names of input proteins were uploaded using H. sapiens as the organism 209 for background list.

210 To illustrate overlap of proteins from the high glucose and the glycolysis inhibition treatment 211 groups (Fig3a) that fulfil criteria (a) p-value $<0.05$ and (b) quantification with $\geq 2$ unique 212 peptides from high glucose and glycolysis inhibition group and (c) a $\geq 1.33$-fold or $\leq 0.75$-fold 213 differential abundance level in these both groups in comparison to the normoglycemic group, we 214 employed BioVenn (Tim Hulsen, http://www.biovenn.nl) (Hulsen, et al., 2008).

215 For generation of the network of proteins (Fig3b), we used STRING (Search Tool for the 216 Retrieval of Interacting Genes/ Proteins, version 11.0, STRING consortium 2017, https://string217 db.org) which belongs to the ELIXIR's Core Data Resources (Szklarczyk et al. 2019). STRING 
218 was employed using "highest confidence" regarding the interaction score, with a score limit of 2190.9 describing the approximate probability that a predicted link exists between two proteins in 220 the same metabolic map in the KEGG database (release 90.1). Additionally, disconnected nodes 221 were hidden while the thickness of the connection lines indicates the strength of the data support. 222 All of the presented connection lines reaching the maximum thickness indicated the maximal 223 strength of the data support. As input format we chose gene names and H. sapiens as organism.

\section{Porcine neuroretinal organotypic explant cultures}

225 Healthy adult porcine eyes were provided by a local abattoir, removed from the animals within 226 five minutes after death and kept on ice in $\mathrm{CO}_{2}$-independent medium until preparation was 227 completed within three hours. One explant was dissected per eye, and all of the eyes were 228 obtained from different animals. Neuroretinal explants were dissected as described (Taylor et al. 229 2014) with some modifications. The opened eyecup was filled with prewarmed $\mathrm{CO}_{2}$-independent 230 medium and the neuroretina was carefully removed from retinal pigment epithelium by very 231 softly flushing medium between the two structures. To ensure correct orientation concerning 232 retinal topography, neuroretina was properly teased back into the original position with a 233 forceps. We carefully excised circular tissue pieces of $6 \mathrm{~mm}$ diameter with a biopsy punch (PFM 234 Medical, Köln, Germany) from the dorso-nasal position of the optic disc (Fig4).

235 Free floating neuroretinal pieces were carefully collected with the upper (large) end of a pasteur 236 pipette connected to a pasteur pipette bulb at the broken lower end and positioned onto $0.4 \mu \mathrm{m}$ 237 culture plate inserts (Thermo Fisher Scientific, Roskilde, Denmark) with the inner limiting 238 membrane side facing the insert membrane and thus providing inner retinal support (Taylor et al. 239 2014). Inserts were placed in six well plates filled with1.5ml RPMI 1640 medium (Thermo 240 Fisher Scientific, Ulm, Germany) containing $11 \mathrm{mM}$ glucose and supplemented with $10 \%$ fetal 241 calf serum and $0.2 \%$ Penicillin-Streptomycin and respective treatment additives. A drop of 242 medium was placed at the air interface on top of each explant and explants were incubated at 37 $243{ }^{\circ} \mathrm{C}$ in $5 \% \mathrm{CO}_{2}$.

\section{Treatment of explants}

245 Treatment started immediately after dissection. During the 72 hours of treatment, culture 246 medium in the chamber below the explant as well as the drop placed on top of the explant was 247 replaced every 24 hours. The normoglycemic group $(n=4$ biological replicates, represented by 248 explants from different eyes and animals) was maintained with a glucose level of $11 \mathrm{mM}$ as 
249 included in RPMI 1640 medium. The hyperglycemic group ( $\mathrm{n}=4$ biological replicates) was 250 cultured under high glucose conditions containing $70 \mathrm{mM}$ glucose due to addition of sterile 251 filtered D-glucose. This concentration was defined in consideration of the factor of 6.25 between 252 normoglycemic and high glucose group that was employed in the isolated RMG cell experiment 253 before (5.6 mM normoglycemic, $35 \mathrm{mM}$ high glucose, 6.25 fold increase). To investigate an 254 osmotic effect independent of the metabolic effects of glucose ( $\mathrm{Yu}$ et al. 2017), we included an 255 256 257 258 osmotic control group ( $\mathrm{n}=4$ biological replicates) containing $11 \mathrm{mM}$ of glucose and $59 \mathrm{mM}$ Dmannose (Millipore Sigma, Darmstadt, Germany) to achieve the same osmolarity like in the high glucose group. Explants representing the glycolysis inhibition group ( $\mathrm{n}=4$ biological replicates) were cultured with levels of $11 \mathrm{mM}$ glucose with addition of $40 \mathrm{mM}$ 2-DG within the last 24 of the 72 hours. This concentration was determined to achieve an about 3.5-fold overdose of 2-DG compared to glucose similar to the RMG experiment (which was $20 \mathrm{mM}$ 2-DG with $5.6 \mathrm{mM}$ glucose).

264

265

266

267

268

269

270

271

272

273

274

275

276

277 278 Germany) and stored at $-20^{\circ} \mathrm{C}$.

\section{Immunohistochemistry of explants}

After 72 hours of treatment, explants were fixated, dehydrated and embedded for cryo sectioning adapted from a protocol provided by Linnea Taylor, Ophthalmology Department, Medical Faculty, Lund University (Taylor et al. 2014). Briefly, prechilled $\left(4{ }^{\circ} \mathrm{C}\right)$ paraformaldehyde (PFA) 4\% (BosterBio, Pleasanton, USA) was drop-wise added to explants still adherent to the insert membrane over a time period of minimal five minutes until they were completely covered with PFA and fixation continued for three hours at $4{ }^{\circ} \mathrm{C}$. PFA was removed and explants were washed three times with prechilled phosphate buffer (PB; $0.1 \mathrm{M}, \mathrm{pH}$ 7.4). Then each explant was excised with $8 \mathrm{~mm}$ biopsy punch (PFM Medical, Köln, Germany) and transferred with the membrane to a flat biopsy capsules (Cell Path, Newton, UK). Cryopreservation was performed by incubating in PB-containing 15\% sucrose (Millipore Sigma, Darmstadt, Germany) for four hours followed by incubation in PB-containing 30\% sucrose overnight at ambient temperature. Explants were taken from the capsule, moved to $12 \mathrm{~mm}$ x $8 \mathrm{~mm}$ embedding molds (Polyscienes, Niles, USA), cryo-embedded together with the membrane in OCT medium (Thermo Fisher Scientific, Ulm, Germany) and stored at $-80^{\circ} \mathrm{C}$. Cryo-sections were collected from the center parts of each

277 explant at $18 \mu \mathrm{m}$ thickness, mounted on coated slides (Superfrost Plus, Thermo Scientific, Ulm, 
279 Slides were defrosted at room temperature for 20 minutes and washed with PB trice for ten 280 minutes each. To avoid unspecific antibody binding, sections were blocked in Tris-buffered 281 saline containing 0.1\% Tween 20 (Serva, Heidelberg, Germany, TBS-T), 1\% bovine serum 282 albumin (Biomol, Hamburg, Germany) and 5\% goat serum (Abcam, Berlin, Germany) for one 283 hour at ambient temperature. Sections were then co-incubated overnight at $4{ }^{\circ} \mathrm{C}$ with rabbit anti284 SPP1 (1:250, OriGene, Herford, Germany) and mouse anti-vimentin (1:50, Millipore Sigma, 285 Darmstadt, Germany) diluted in TBS-T with 1\% bovine serum albumin and 5\% goat serum, 286 followed by co-incubation with goat anti-rabbit IgG AlexaFluor 488 and goat anti-mouse IgG 287 AlexaFluor 568 (each 1:500, Thermo Fisher Scientific, Ulm, Germany) for 90 minutes at 288 ambient temperature. Between primary and secondary resp. after secondary antibody incubations 289 sections were washed three times each with PB for 10 minutes. After counterstaining with 290 Hoechst (1:10,000, Thermo Fisher Scientific, Ulm, Germany) for 10 minutes at ambient 291 temperature, coverslips were mounted in aqueous FluorSave cover medium (Thermo Fisher 292 Scientific, Ulm, Germany) and photographed on a Leica DMi8 microscope with the HC PL APO 293 40x/0.95 DRY objective lens. Filter cubes for GFP, Texas Red and DAPI detections were used 294 (JH Technologies). All images were captured using a Leica DFC365 FX camera, and constant 295 296 settings for gain and exposure time were maintained for all sections within an experimental setup. Images were processed by the Leica Application Suite LASX (version 3.03, Leica). As

297 298 299 300 301 302 303 304 305 306 307

control, three sections per group from different explants were stained under equal conditions with anti-rabbit-IgG (1:1,000, Abcam, Berlin, Germany) instead of anti-SPP1; no unspecific labeling was observed.

\section{Results}

Overrepresentation of ECM-associated biological processes in RMG under diabetic culture conditions

We isolated primary porcine RMG from pig eyes, applied the diabetes short term treatment and investigated proteome-wide changes applying a mass spectrometric work flow (Fig. 1). A total of 2744 proteins quantified with $\geq 2$ unique peptides were detected across in all treatment groups and replicates (Supplemental table 1).

In the high glucose treatment group, a total of 464 proteins were significantly differently abundant compared to the normoglycemic control group (Fig. 2a, protein-dots above the

Peer] reviewing PDF | (2020:12:56055:1:1:NEW 15 Mar 2021) 
310 reference line for $\mathrm{p}$-value cut-off, supplemental table 2), thereof 103 proteins met the additional

311 criterion of fold change cut-off (Fig. 2a, colored protein-dots beyond both reference lines for fold

312 change cut-off). Pathway enrichment analysis of these 103 proteins revealed the top ten

313 biological processes that were significantly enriched with a functional relationship to the input

314 proteins (Fig. 2b). The three highest ranking pathways are strongly associated with extracellular

315 matrix function, namely "collagen fibril organization", "extracellular matrix organization" and

316 "extracellular structure organization" (Fig. 2b, supplemental table 3). Accordingly, among the

317 changed proteins were 19 members of the ECM (supplemental table 2, according to the human

318 matrisome database, http://matrisomeproject.mit.edu (Naba, et al., 2016); Fig2a, labeled with

319 gene symbols) of which the majority (16) showed lower abundance levels in the high glucose

320 treatment group compared to the normoglycemic control group.

321 In the glycolysis inhibition treatment group, altogether 800 proteins exhibited significant changes

322 in abundance levels compared to the normoglycemic control group (Fig. 2c, protein-dots above

323 the reference line for p-value cut-off, supplemental table 4;), of which 240 proteins additionally

324 met the fold change cut-off (Fig. 2c, colored protein-dots beyond both reference lines for fold

325 change cut-off). Again, pathway enrichment analysis of these 240 proteins pointed to an

326 overrepresentation of proteins belonging to the context of extracellular matrix, which was

327 demonstrated by the three most significantly overrepresented biological processes (Fig. 2d,

328 namely "extracellular structure organization", "collagen fibril organization" and "extracellular

329 matrix organization"; supplemental table 5). Correspondingly, there were 34 ECM members

330 among the input proteins (supplemental table 4, according to the human matrisome database;

331 Fig. 2c labeled with gene symbols), with the majority (28) once again showing lower abundance

332 levels in the glycolysis inhibition treatment group compared to the normoglycemic control

333 group. Interestingly, secreted phosphoprotein 1 (SPP1) appeared in both diabetes-like conditions 334 as one of the top changed candidates.

335 Downregulation of ECM organization and cell adhesion processes under diabetic culture 336 conditions

337 Both diabetic treatments caused a similar pattern of highly overrepresented processes when

338 compared to the normoglycemic control. Hence, we aimed to clarify if this could be linked to a

339 similar alteration in abundance level of particular proteins or groups of proteins. There were

340 differences in the proteins with changed abundance between diabetic conditions, but 65 of all 
341 differently abundant proteins overlapped (Fig. 3a). We concluded that these 65 proteins have

342 fundamental roles in RMG metabolism, since they reacted consistently to different changes

343 applied to glucose metabolism by our experimental treatments. We next performed a protein

344 enrichment analyses with STRING, applying strict evaluation criteria to investigate the major

345 functions of these 65 proteins. These analyses confirmed enriched interactions of proteins of the

346 biological process "ECM organization" and additionally defined the biological process "cell

347 adhesion" (Fig. 3b, according to the Gene Ontology term of biological processes). Sixty-two of

34865 proteins changed their abundance in the same way, so either decreasing (39 proteins) or

349 increasing (23 proteins) their abundance levels in both treatment groups compared to the control

350 group. All of the proteins belonging to the "ECM organization" and "cell adhesion" biological

351 process in the STRING network are proteins with decreased abundance in both treatment groups

352 compared to the normoglycemic control group. That points to a downregulation of both

353 processes in high glucose and glycolysis inhibition treatment.

354 Some of the candidates have functions in ECM organization as well as in cell adhesion. Among 355 them is SPP1 (Fig. 3b), that interacts in both pathways with collagen- $\alpha$-chains, fibronectin and

356 the alpha integrins ITGA5, ITGA8, ITGAL (Fig. 3b, supplemental tables 2 and 4). We therefore

357 decided to further investigate SPP1 in our hyperglycemia model.

358 RMG-associated SPP1 expression pattern selectively diminished in retinal explants under 359 diabetic culture conditions

360 SPP1 was consistently decreased in both diabetic conditions and belongs to several

361 overrepresented biological processes in the dataset. Earlier, SPP1 was shown to play a key role

362 in RMG mediated pathogenesis in an inflammatory eye disease (Deeg, et al., 2011). Further,

363 SPP1 was recently found to be secreted by porcine RMG in vitro, exhibiting a survival-

364 promoting effect on porcine retinal ganglion cells in vitro (Ruzafa et al. 2018b).

365 To analyze SPP1 in the context of the retinal network of RMG cells, we next used porcine retinal

366 explants as a model. We took pieces from the visual streak (Fig. 4a), a region of the porcine

367 retina which is characterized by very high densities of cones and ganglion cells, in a ratio that is

368 also found in humans (Garca, et al., 2005, Hendrickson and Hicks, 2002). Explants were cultured

369 on the fluid/air interface on a polycarbonate membrane (Fig. 4b). In reference to the isolated

370 porcine RMG, short term diabetic treatment was applied. An osmotic control group was included 
371 to determine the osmotic effect independently of the effect of high glucose or glycolysis

372 inhibition to the explants (Fig. 4b).

373 Since SPP1 expression patterns in porcine retina were not reported so far to our knowledge, we

374 initially examined it's distribution by immunohistochemical fluorescence staining of porcine

375 retinal organotypic explant cultures after three days in vitro under normoglycemic conditions 376 (Fig. 5).

377 SPP1 was detected at the level of photoreceptor outer segments (POS) and in the inner

378 neuroretina in the area of the ganglion cell layer (GCL) and nerve fiber layer (NFL) (Fig. 5, top, 379 left panel). Vimentin was used as marker for RMG and its characteristic RMG morphology (Fig.

3805 , top, mid panel). Double labeling of SPP1 with vimentin revealed co-localization along RMG

381 processes and endfeet in the inner neuroretina (Fig. 5, top, right panel). This co-localization in

382 the area of the GCL and NFL suggests RMG-associated SPP1 expression also in the ex vivo 383 retinal system.

384 We then aimed to investigate if SPP1 expression in the ex vivo retinal system is downregulated in 385 response to diabetic culture conditions. In all four conditions, vimentin was characteristically 386 expressed in RMG processes throughout the inner retina from the endfeet that build the inner 387 limiting membrane to the outer plexiform layer (Fig. 5, mid panels, vimentin red). In osmotic 388 control and in both diabetic conditions (high glucose and glycolysis inhibitions), the RMG389 associated SPP1 diminished in comparison to the normoglycemic cultured explants (Fig. 5, left 390 panels, SPP1 green; overlay with vimentin staining: right panels). Interestingly, SPP1 staining at 391 the level of photoreceptor outer segments remained unaltered in all conditions, indicating a 392 specific RMG response.

393

394 Discussion

395 Diabetes mellitus is currently one of the major health concerns with nearly half a billion people 396 affected worldwide and a permanently increasing prevalence (Saeedi et al. 2019). Chronic

397 hyperglycemia and its consequences are the main issues of diabetes mellitus (Renner et al. 2013).

398 Diabetic retinopathy as a microvascular complication is also one of the fastest growing diseases 399 worldwide, leading to blindness and greatly impairs quality of life in affected patients (Harding 400 et al. 2019). To date, the mechanisms leading to retinal damage are not well understood. 
401 Appropriate animal models to address the pathogenesis of diabetic retinopathy are scarce to date, 402 because traditional animal models in rodents lack translational value because of significant 403 differences in physiological, anatomical and immunological aspects. The pig recently evolved as 404 a favorable model for diabetes given the marked similarity with the humans with respect to the 405 developed pathophysiological features (Cole \& Florez 2020; Kleinwort et al. 2017; Renner et al. 406 2020; Renner et al. 2013). RMG is the most important glial cell in the retina where it plays a 407 central role (Bringmann et al. 2006; Bringmann \& Wiedemann 2012) in the pathology of DR, 408 RMG seem to be fundamentally involved. However, the molecular mechanisms of pathological 409 changes to and initiated by this specific cell type are not well understood (McDowell et al. 2018).

410 Recently, a proteomic study in diabetic patients demonstrated retinol binding protein-3 to control 411 glucose transport in RMG and thus development of DR (Yokomizo et al. 2019). This study 412 highlights not only the successful application of proteomic techniques to elucidate molecular 413 pathomechanisms in DR, but also the important role of RMG in pathologic processes of diabetic 414 retina. To the best of our knowledge proteome-wide responses of RMG to diabetes-like 415 metabolic situations have not been studied before and by using a comprehensive, differential 416 proteomic analysis in our primary RMG cell culture model (Hauck et al. 2003), we were able to 417 investigate specific mechanisms attributed to this particular cell type. Short term treatment in cell 418 culture allowed to shed light on the mechanisms underlying the initial phases of disturbed 419 glucose metabolism. Applying a differential proteome analysis approach, we observed highly 420 significant proteome changes in cells cultured under two different treatment regimens designed 421 to mimic fluctuating glucose levels naturally occurring in diabetes. A total of 65 proteins showed 422 a significant differential expression upon both diabetes-like conditions. A pathway enrichment 423 analysis of the proteins with altered abundance revealed an enrichment of ECM associated 424 pathways, with a hinted downregulation of ECM organization and cell adhesion. Decreased 425 production of ECM proteins under diabetic conditions could explain our previous finding of the 426 disruption of the inner limiting membrane (ILM) in a diabetic pig model resulting in 427 accumulation of hard exudates and cotton-wool spots, which are common features of 428 proliferative neovascularization (Kleinwort et al. 2017).

429 The ILM is a thin sheet composed of ECM proteins at the inner surface of the retina closely 430 connected to RMG endfeet (Syrbe et al. 2018). There is evidence that human RMG produce 431 ECM proteins suggesting their role in the ILM assembly (Ponsioen et al. 2008). The present data 
432 point toward a remodeling of ECM proteins which potentially destabilizes the ILM thereby 433 impairing its integrity in DR. Further support to our hypothesis on RMG involvement in de434 stabilizing ILM is provided by the observed downregulation of integrins, which are key factors 435 for the correct assembly and function of ILM (Halfter et al. 2008).

436 Since the retina comprises a highly sophisticated neuronal network of many specialized cells in 437 close interaction, we aimed to confirm our findings in a model which closely resembles in vivo 438 conditions. Hence we established porcine organotypic retinal explants cultures which preserve 439 the cellular architecture and functional interactions physiologically present in the tissue hence 440 providing an adequate and comprehensive model of the retina (Schnichels et al. 2019).

441 In this study, porcine retinal explants were excised from the visual streak, a region of the porcine 442 retina which is characterized by high densities of cones and ganglion cells (Garca et al. 2005; 443 Hendrickson \& Hicks 2002). The region dorso-nasal the optic disc within the visual streak 444 contains the highest cone numbers and thus resembles the human macula (Hendrickson \& Hicks 445 2002; Kleinwort et al. 2017). We therefore examined a retinal region with a similar cellular 446 distribution like the human macula to use the advantage of the porcine model to learn about 447 human macula diseases.

448 We identified SPP1 (synonym osteopontin) as a major changed candidate in our study. SPP1 is 449 an interesting molecule with pleiotropic functions (Ruzafa et al. 2018a). The function in the 450 retina is not entirely clear to date (Ruzafa et al. 2018a). SPP1 influences cell survival and 451 attachment, cell migration, inflammation, migration and homeostasis after injury (Ruzafa et al. 452 2018a). In this study, SPP1 disappeared significantly and rapidly in short term hyperglycemic 453 conditions in cell culture models and likewise in organotypic cultures. Although the exact 454 implications for diabetic retinopathy remain open at this point, the loss of a SPP1, which has 455 been described to rescue photoreceptors and to be induced by neuroprotective factor GDNF (Del 456 Rio et al. 2011), is an important finding of our study and deserves further investigation in our 457 opinion. Interestingly, the loss of protein abundance was not limited to SPP1, but was also 458 observed for its known interactors, the integrins (Yang et al. 2019).

459 Here, among other integrins, ITGB1 was downregulated under simulated diabetic conditions. 460 Conditional deletion of ITBG1 in mouse brain leads to partial gliosis of astrocytes (Robel et al. 461 2009). Gliosis is generally considered as a protective response of glial cells to harmful insults 462 (Bringmann et al. 2006; Burda \& Sofroniew 2014) and occurs in RMG probably in an early stage 
463 of DR (Vujosevic et al. 2015). However, in contrast to the observed downregulation of ITGB1, 464 GFAP and vimentin, two prototype markers of gliosis, were not changed by modifying glucose 465 levels in RMG (see supplemental table 1). Therefore, we speculate that changes in ECM proteins 466 related to gliosis could be triggered by high glucose treatment even before upregulation of 467 intermediate filament proteins (Liu et al. 2016), as well established marker for reactive RMG 468 gliosis (Subirada et al., 2018).

469 Our results may indicate an involvement of RMG in the neurodegenerative processes in early

470 DR, because SPP1 protein was consistently found less abundant in cultured RMG under diabetic 471 treatment conditions. To date, we do not know the molecular mechanism leading to decrease in 472 SPP1 in these conditions. Potential effects of hyperglycemia in diabetes range from specific 473 metabolic effects, through non-enzymatic glycation, glycoxidation, lipoxidation and osmotic 474 stress (Yu et al. 2007). In our cell culture environment, mannose was applied to specifically 475 induce osmotic effects in the explants and to distinguish them from the other hyperglycemia476 induced mechanisms (Yu et al. 2007). Since the observed effects on the abundance of SPP1 also 477 occurred in the osmose control group, we speculate that alterations in the SPP1 protein levels 478 might result from a multi-causative osmotic stress. This report is the first to describe a RMG 479 specific SPP1 regulation in a high glucose and hyperosmolar state. Since the hyperglycemic 480 hyperosmolar state is a serious acute metabolic complication of diabetes mellitus (Umpierrez \& 481 Korytkowski 2016), further experiments should unravel potential relations of hyperglycemic and 482 osmotic conditions that induce decrease in SPP1 levels.

483 Interestingly, it has been shown that supplemented SPP1 inhibits experimentally induced osmotic 484 swelling in ex vivo rat RMG (Wahl et al. 2013). Glial swelling is involved in pathology of 485 diabetic macula edema, a severe complication of DR (Coughlin et al. 2017; Daruich et al. 2018; 486 Graue-Hernandez et al. 2020; Wahl et al. 2013). We speculate that a decrease in SPP1 expression 487 level might contribute to RMG swelling in diabetic macular edema. In the retina of diabetic pigs 488 we observed a central swelling of the NFL (Kleinwort et al. 2017). It is reasonable to believe that 489 decreased expression of SPP1 could account for the swelling of glial cells as well as the 490 surrounding structures.

491 Noteworthy is the neuroprotective function of SPP1 in retina. In the porcine model, a pro492 survival effect of SPP1 on in vitro retinal ganglion cells (Ruzafa et al. 2018b) and on 493 photoreceptors (Del Rio et al. 2011) has been described. We believe that under diabetic 
494 conditions, reduced expression of RMG-associated SPP1 might contribute to severe neuronal 495 damage in DR in vivo. Investigating whether neuronal survival in porcine retinal explants is 496 affected by RMG-associated SPP1 expression will be the focus of our future studies.

497 Interestingly, we have also previously found a significant downregulation of RMG-associated 498 SPP1 expression in retinas of horses with uveitis (Deeg et al. 2011). Collectively, these data 499 point to SPP1 being generally involved in retinal pathology.

500

\section{Conclusions}

502 Our findings suggest an initial involvement of RMG in DR. Perturbation with glucose levels 503 significantly alters many ECM proteins in RMG suggesting profound ECM remodeling; secreted 504 phosphoprotein 1 (SPP1) being one of these. Given the previously described neuroprotective role 505 of SPP1 and its inhibitory role on RMG cell swelling, SPP1 could hold potential as candidate 506 molecule for developing treatment strategies. Modulating SPP1 levels, as well as other 507 components of the ECM could help to prevent neurodegeneration in DR and RMG might 508 represent promising targets in such circumstances.

509

\section{Acknowledgements}

511 We would like to thank Linnea Taylor from the Ophthalmology Department, Medical Faculty, 512 Lund University for her help in establishing the porcine retinal explants in our lab and critical 513 discussions.

514

\section{Funding Information}

516 This work was funded by grants from the Deutsche Forschungsgemeinschaft: HAU 6014/5-1 (to 517 Stefanie M Hauck) und DE 719/7-1 (to Cornelia A Deeg) in the priority program SPP 2127. 


\section{References}

521

522

523

524

525

526

527

528

529

530

531

532

533

534

535

536

537

538

539

540

541

542

543

544

545

546

547

548

549

550

551

552

553

554

555

556

557

558

559

560

561

562

563

564

565

566

567

Bringmann A, Pannicke T, Grosche J, Francke M, Wiedemann P, Skatchkov SN, Osborne NN, and Reichenbach A. 2006. Muller cells in the healthy and diseased retina. Progress in Retinal and Eye Research 25:397-424. 10.1016/j.preteyeres.2006.05.003

Bringmann A, and Wiedemann P. 2012. Müller Glial Cells in Retinal Disease. Ophthalmologica 227:1-19. 10.1159/000328979

Burda JE, and Sofroniew MV. 2014. Reactive gliosis and the multicellular response to CNS damage and disease. Neuron 81:229-248. 10.1016/j.neuron.2013.12.034

Chertov AO, Holzhausen L, Kuok IT, Couron D, Parker E, Linton JD, Sadilek M, Sweet IR, and Hurley JB. 2011. Roles of glucose in photoreceptor survival. J Biol Chem 286:3470034711. 10.1074/jbc.M111.279752

Cole JB, and Florez JC. 2020. Genetics of diabetes mellitus and diabetes complications. Nature Reviews Nephrology. 10.1038/s41581-020-0278-5

Coughlin BA, Feenstra DJ, and Mohr S. 2017. Muller cells and diabetic retinopathy. Vision Res 139:93-100. 10.1016/j.visres.2017.03.013

Daruich A, Matet A, Moulin A, Kowalczuk L, Nicolas M, Sellam A, Rothschild P-R, Omri S, Gélizé E, Jonet L, Delaunay K, De Kozak Y, Berdugo M, Zhao M, Crisanti P, and BeharCohen F. 2018. Mechanisms of macular edema: Beyond the surface. Progress in Retinal and Eye Research 63:20-68. https://doi.org/10.1016/j.preteyeres.2017.10.006

Deeg CA, Eberhardt C, Hofmaier F, Amann B, and Hauck SM. 2011. Osteopontin and fibronectin levels are decreased in vitreous of autoimmune uveitis and retinal expression of both proteins indicates ECM re-modeling. PLoS One 6:e27674.

10.1371/journal.pone.0027674

Del Rio P, Irmler M, Arango-Gonzalez B, Favor J, Bobe C, Bartsch U, Vecino E, Beckers J, Hauck SM, and Ueffing M. 2011. GDNF-induced osteopontin from Muller glial cells promotes photoreceptor survival in the Pde6brd1 mouse model of retinal degeneration. Glia 59:821-832. 10.1002/glia.21155

Ducos C, Rigo M, Larroumet A, Delyfer M-N, Korobelnik J-F, Monlun M, Foussard N, Poupon P, Haissaguerre M, Blanco L, Mohammedi K, and Rigalleau V. 2020. Diabetic retinopathy in well-controlled type 2 diabetes: role of glycaemic memory. Diabetes \& Metabolism. https://doi.org/10.1016/j.diabet.2020.03.005

Fu S, Dong S, Zhu M, Sherry DM, Wang C, You Z, Haigh JJ, and Le YZ. 2015. Muller Glia Are a Major Cellular Source of Survival Signals for Retinal Neurons in Diabetes. Diabetes 64:3554-3563. 10.2337/db15-0180

Garca M, Ruiz-Ederra J, Hernandez-Barbachano H, and Vecino E. 2005. Topography of pig retinal ganglion cells. J Comp Neurol 486:361-372. 10.1002/cne.20516

Giese IM, Schilloks MC, Degroote RL, Weigand M, Renner S, Wolf E, Hauck SM, and Deeg CA. 2020. Chronic Hyperglycemia Drives Functional Impairment of Lymphocytes in Diabetic INS (C94Y) Transgenic Pigs. Front Immunol 11:607473. 10.3389/fimmu.2020.607473

Graue-Hernandez EO, Rivera-De-La-Parra D, Hernandez-Jimenez S, Aguilar-Salinas CA, Kershenobich-Stalnikowitz D, and Jimenez-Corona A. 2020. Prevalence and associated risk factors of diabetic retinopathy and macular oedema in patients recently diagnosed with type 2 diabetes. BMJ open ophthalmology 5:e000304-e000304. 10.1136/bmjophth2019-000304

Grosche A, Hauser A, Lepper MF, Mayo R, von Toerne C, Merl-Pham J, and Hauck SM. 2016. The Proteome of Native Adult Muller Glial Cells From Murine Retina. Mol Cell Proteomics 15:462-480. 10.1074/mcp.M115.052183

Peer] reviewing PDF | (2020:12:56055:1:1:NEW 15 Mar 2021) 
568

569

570

571

572

573

574

575

576

577

578

579

580

581

582

583

584

585

586

587

588

589

590

591

592

593

594

595

596

597

598

599

600

601

602

603

604

605

606

607

608

609

610

611

612

613

614

615

616
Halfter W, Dong S, Dong A, Eller AW, and Nischt R. 2008. Origin and turnover of ECM proteins from the inner limiting membrane and vitreous body. Eye (Lond) 22:1207-1213. 10.1038/eye.2008.19

Harding JL, Pavkov ME, Magliano DJ, Shaw JE, and Gregg EW. 2019. Global trends in diabetes complications: a review of current evidence. Diabetologia 62:3-16. 10.1007/s00125-018-4711-2

Hauck SM, Gloeckner CJ, Harley ME, Schoeffmann S, Boldt K, Ekstrom PA, and Ueffing M. 2008. Identification of paracrine neuroprotective candidate proteins by a functional assay-driven proteomics approach. Molecular \& Cellular Proteomics : MCP 7:13491361. 10.1074/mcp.M700456-MCP200

Hauck SM, Suppmann S, and Ueffing M. 2003. Proteomic profiling of primary retinal Muller glia cells reveals a shift in expression patterns upon adaptation to in vitro conditions. Glia 44:251-263.

Hauck SM, von Toerne C, and Ueffing M. 2014. The neuroprotective potential of retinal Muller glial cells. Lacrimal Gland, Tear Film, and Dry Eye Syndromes 3: Basic Science and Clinical Relevance, Pts a \& B 801:381-387. 10.1007/978-1-4614-3209-8_48

Hendrickson A, and Hicks D. 2002. Distribution and density of medium- and short-wavelength selective cones in the domestic pig retina. Experimental eye research 74:435-444. 10.1006/exer.2002.1181

Kadlubowska J, Malaguarnera L, Waz P, and Zorena K. 2016. Neurodegeneration and Neuroinflammation in Diabetic Retinopathy: Potential Approaches to Delay Neuronal Loss. Current Neuropharmacology 14:831-839. 10.2174/1570159x14666160614095559

Kanwar M, and Kowluru RA. 2009. Role of glyceraldehyde 3-phosphate dehydrogenase in the development and progression of diabetic retinopathy. Diabetes 58:227-234. 10.2337/db08-1025

Kleinwort KJH, Amann B, Hauck SM, Hirmer S, Blutke A, Renner S, Uhl PB, Lutterberg K, Sekundo W, Wolf E, and Deeg CA. 2017. Retinopathy with central oedema in an INS (C94Y) transgenic pig model of long-term diabetes. Diabetologia 60:1541-1549. 10.1007/s00125-017-4290-7

Leasher JL, Bourne RR, Flaxman SR, Jonas JB, Keeffe J, Naidoo K, Pesudovs K, Price H, White RA, Wong TY, Resnikoff S, Taylor HR, and Vision Loss Expert Group of the Global Burden of Disease S. 2016. Global Estimates on the Number of People Blind or Visually Impaired by Diabetic Retinopathy: A Meta-analysis From 1990 to 2010. Diabetes Care 39:1643-1649. 10.2337/dc15-2171

Lepper MF, Ohmayer U, von Toerne C, Maison N, Ziegler AG, and Hauck SM. 2018. Proteomic Landscape of Patient-Derived CD4+ T Cells in Recent-Onset Type 1 Diabetes. Journal of Proteome Research 17:618-634. 10.1021/acs.jproteome.7b00712

Mathebula SD. 2018. Biochemical changes in diabetic retinopathy triggered by hyperglycaemia: A review. African Vision and Eye Health 77:7. 10.4102/aveh.v77i1.439

Mattugini N, Merl-Pham J, Petrozziello E, Schindler L, Bernhagen J, Hauck SM, and Gotz M. 2018. Influence of white matter injury on gray matter reactive gliosis upon stab wound in the adult murine cerebral cortex. Glia 66:1644-1662. 10.1002/glia.23329

Mazade RE, and Eggers ED. 2020. Inhibitory components of retinal bipolar cell receptive fields are differentially modulated by dopamine D1 receptors. Visual Neuroscience 37:E01. $10.1017 /$ S0952523819000129

McDowell RE, Barabas P, Augustine J, Chevallier O, McCarron P, Chen M, McGeown JG, and Curtis TM. 2018. Muller glial dysfunction during diabetic retinopathy in rats is reduced by the acrolein-scavenging drug, 2-hydrazino-4,6-dimethylpyrimidine. Diabetologia 61:2654-2667. 10.1007/s00125-018-4707-y 
617

618

619

620

621

622

623

624

625

626

627

628

629

630

631

632

633

634

635

636

637

638

639

640

641

642

643

644

645

646

647

648

649

650

651

652

653

654

655

656

657

658

659

660

661

662

663

664

665

666

Menduni F, Davies LN, Madrid-Costa D, Fratini A, and Wolffsohn JS. 2018. Characterisation of the porcine eyeball as an in-vitro model for dry eye. Contact Lens and Anterior Eye 41:13-17. 10.1016/j.clae.2017.09.003

Mesquida M, Drawnel F, and Fauser S. 2019. The role of inflammation in diabetic eye disease. Seminars in Immunopathology 41:427-445. 10.1007/s00281-019-00750-7

Mi XS, Yuan TF, Ding Y, Zhong JX, and So KF. 2014. Choosing preclinical study models of diabetic retinopathy: key problems for consideration. Drug Design, Development and Therapy 8:2311-2319. 10.2147/DDDT.S72797

Ogurtsova K, da Rocha Fernandes JD, Huang Y, Linnenkamp U, Guariguata L, Cho NH, Cavan D, Shaw JE, and Makaroff LE. 2017. IDF Diabetes Atlas: Global estimates for the prevalence of diabetes for 2015 and 2040. Diabetes Research and Clinical Practice 128:40-50. 10.1016/j.diabres.2017.03.024

Ola MS, Berkich DA, Xu Y, King MT, Gardner TW, Simpson I, and LaNoue KF. 2006. Analysis of glucose metabolism in diabetic rat retinas. Am J Physiol Endocrinol Metab 290:E1057-1067. 10.1152/ajpendo.00323.2005

Poitry-Yamate C, and Tsacopoulos M. 1991. Glial (Muller) cells take up and phosphorylate [3H]2-deoxy-D-glucose in mammalian retina. Neurosci Lett 122:241-244. 10.1016/03043940(91)90868-t

Ponsioen TL, van Luyn MJ, van der Worp RJ, Pas HH, Hooymans JM, and Los LI. 2008. Human retinal Muller cells synthesize collagens of the vitreous and vitreoretinal interface in vitro. Molecular Vision 14:652-660.

Renner S, Blutke A, Clauss S, Deeg CA, Kemter E, Merkus D, Wanke R, and Wolf E. 2020. Porcine models for studying complications and organ crosstalk in diabetes mellitus. Cell and Tissue Research 380:341-378. 10.1007/s00441-019-03158-9

Renner S, Braun-Reichhart C, Blutke A, Herbach N, Emrich D, Streckel E, Wunsch A, Kessler B, Kurome M, Bahr A, Klymiuk N, Krebs S, Puk O, Nagashima H, Graw J, Blum H, Wanke R, and Wolf E. 2013. Permanent neonatal diabetes in INS(C94Y) transgenic pigs. Diabetes 62:1505-1511. 10.2337/db12-1065

Robel S, Mori T, Zoubaa S, Schlegel J, Sirko S, Faissner A, Goebbels S, Dimou L, and Gotz M. 2009. Conditional deletion of beta1-integrin in astroglia causes partial reactive gliosis. Glia 57:1630-1647. 10.1002/glia.20876

Ruzafa N, Pereiro X, Aspichueta P, Araiz J, and Vecino E. 2018a. The Retina of Osteopontin deficient Mice in Aging. Mol Neurobiol 55:213-221. 10.1007/s12035-017-0734-9

Ruzafa N, Pereiro X, Lepper MF, Hauck SM, and Vecino E. 2018b. A Proteomics Approach to Identify Candidate Proteins Secreted by Muller Glia that Protect Ganglion Cells in the Retina. PROTEOMICS 18:e1700321. 10.1002/pmic.201700321

Saeedi P, Petersohn I, Salpea P, Malanda B, Karuranga S, Unwin N, Colagiuri S, Guariguata L, Motala AA, Ogurtsova K, Shaw JE, Bright D, Williams R, and Committee IDFDA. 2019. Global and regional diabetes prevalence estimates for 2019 and projections for 2030 and 2045: Results from the International Diabetes Federation Diabetes Atlas, 9(th) edition. Diabetes Res Clin Pract 157:107843. 10.1016/j.diabres.2019.107843

Schnichels S, Kiebler T, Hurst J, Maliha AM, Loscher M, Dick HB, Bartz-Schmidt KU, and Joachim SC. 2019. Retinal Organ Cultures as Alternative Research Models. Altern Lab Anim 47:19-29. 10.1177/0261192919840092

Sorrentino FS, Allkabes M, Salsini G, Bonifazzi C, and Perri P. 2016. The importance of glial cells in the homeostasis of the retinal microenvironment and their pivotal role in the course of diabetic retinopathy. Life Sci 162:54-59. 10.1016/j.Ifs.2016.08.001

Subirada PV, Paz MC, Ridano ME, Lorenc VE, Vaglienti MV, Barcelona PF, Luna JD, and Sanchez MC. 2018. A journey into the retina: Muller glia commanding survival and death. Eur J Neurosci 47:1429-1443. 10.1111/ejn.13965

Peer) reviewing PDF | (2020:12:56055:1:1:NEW 15 Mar 2021) 
667

668

669

670

671

672

673

674

675

676

677

678

679

680

681

682

683

684

685

686

687

688

689

690

691

692

693

694

695

696

697

698

699

700

701

702

703

704

705

706

707

708

709

710

711

712

713

714

715

Syrbe S, Kuhrt H, Gartner U, Habermann G, Wiedemann P, Bringmann A, and Reichenbach A. 2018. Muller glial cells of the primate foveola: An electron microscopical study. Experimental eye research 167:110-117. 10.1016/j.exer.2017.12.004

Szklarczyk D, Gable AL, Lyon D, Junge A, Wyder S, Huerta-Cepas J, Simonovic M, Doncheva NT, Morris JH, Bork P, Jensen LJ, and Mering CV. 2019. STRING v11: protein-protein association networks with increased coverage, supporting functional discovery in genome-wide experimental datasets. Nucleic Acids Res 47:D607-D613. 10.1093/nar/gky1131

Taylor L, Arner K, Taylor IH, and Ghosh F. 2014. Feet on the ground: Physical support of the inner retina is a strong determinant for cell survival and structural preservation in vitro. Invest Ophthalmol Vis Sci 55:2200-2213. 10.1167/iovs.13-13535

Thomas RL, Halim S, Gurudas S, Sivaprasad S, and Owens DR. 2019. IDF Diabetes Atlas: A review of studies utilising retinal photography on the global prevalence of diabetes related retinopathy between 2015 and 2018. Diabetes Research and Clinical Practice 157. 10.1016/j.diabres.2019.107840

Toft-Kehler AK, Skytt DM, Svare A, Lefevere E, Van Hove I, Moons L, Waagepetersen HS, and Kolko M. 2017. Mitochondrial function in Müller cells - Does it matter? Mitochondrion 36:43-51. https://doi.org/10.1016/j.mito.2017.02.002

Umpierrez G, and Korytkowski M. 2016. Diabetic emergencies — ketoacidosis, hyperglycaemic hyperosmolar state and hypoglycaemia. Nature Reviews Endocrinology 12:222-232. 10.1038/nrendo.2016.15

Valdes J, Trachsel-Moncho L, Sahaboglu A, Trifunovic D, Miranda M, Ueffing M, PaquetDurand F, and Schmachtenberg O. 2016. Organotypic retinal explant cultures as in vitro alternative for diabetic retinopathy studies. ALTEX 33:459-464. 10.14573/altex.1603111

Vujosevic S, Micera A, Bini S, Berton M, Esposito G, and Midena E. 2015. Aqueous Humor Biomarkers of Muller Cell Activation in Diabetic Eyes. Invest Ophthalmol Vis Sci 56:3913-3918. 10.1167/iovs.15-16554

Wahl V, Vogler S, Grosche A, Pannicke T, Ueffing M, Wiedemann P, Reichenbach A, Hauck SM, and Bringmann A. 2013. Osteopontin inhibits osmotic swelling of retinal glial (Muller) cells by inducing release of VEGF. Neuroscience 246:59-72. 10.1016/j.neuroscience.2013.04.045

Weigand M, Degroote RL, Amann B, Renner S, Wolf E, Hauck SM, and Deeg CA. 2020. Proteome profile of neutrophils from a transgenic diabetic pig model shows distinct changes. J Proteomics 224.

Wisniewski JR, Zougman A, Nagaraj N, and Mann M. 2009. Universal sample preparation method for proteome analysis. Nat Methods 6:359-362. 10.1038/nmeth.1322

Yang J, Chen C, McLaughlin T, Wang Y, Le Y-Z, Wang JJ, and Zhang SX. 2019. Loss of X-box binding protein 1 in Müller cells augments retinal inflammation in a mouse model of diabetes. Diabetologia 62:531-543. 10.1007/s00125-018-4776-y

Yokomizo H, Maeda Y, Park K, Clermont AC, Hernandez SL, Fickweiler W, Li Q, Wang CH, Paniagua SM, Simao F, Ishikado A, Sun B, Wu IH, Katagiri S, Pober DM, Tinsley LJ, Avery RL, Feener EP, Kern TS, Keenan HA, Aiello LP, Sun JK, and King GL. 2019. Retinol binding protein 3 is increased in the retina of patients with diabetes resistant to diabetic retinopathy. Sci Transl Med 11. 10.1126/scitransImed.aau6627

Yu H, Liu X, and Zhong Y. 2017. The Effect of Osteopontin on Microglia. Biomed Res Int 2017:1879437. 10.1155/2017/1879437

Yu T, Sheu S-S, Robotham JL, and Yoon Y. 2008. Mitochondrial fission mediates high glucoseinduced cell death through elevated production of reactive oxygen species. Cardiovascular research 79:341-351. 10.1093/cvr/cvn104

Peer) reviewing PDF | (2020:12:56055:1:1:NEW 15 Mar 2021) 
716 Yu Y, Li W, Wojciechow B, Jenkins AJ, and Lyons TJ. 2007. Effects of D- and L-Glucose and

717

718

719

720

721

722

723

724

725

726

727

728 Mannitol on Retinal Capillary Cells: Inhibition by Nanomolar Aminoguanidine. American Journal of Pharmacology and Toxicology 2:148-158. 10.3844/ajptsp.2007.148.158

Zhang D, Fei Q, Li J, Zhang C, Sun Y, Zhu C, Wang F, and Sun Y. 2016. 2-Deoxyglucose Reverses the Promoting Effect of Insulin on Colorectal Cancer Cells In Vitro. PLoS One 11:e0151115. 10.1371/journal.pone.0151115

Zhang JZ, Gao L, Widness M, Xi X, and Kern TS. 2003. Captopril inhibits glucose accumulation in retinal cells in diabetes. Investigative Ophthalmology \& Visual Science 44:4001-4005. 10.1167/iovs.02-1193

Zhu ZX, Cai WH, Wang T, Ye HB, Zhu YT, Chi LS, Duan YM, Sun CC, Xuan YH, and Jin LT. 2015. bFGF-Regulating MAPKs Are Involved in High Glucose-Mediated ROS Production and Delay of Vascular Endothelial Cell Migration. PLoS One 10:e0144495-e0144495.

729 10.1371/journal.pone.0144495

730 


\section{Figure 1}

Retinal RMG proteome profiling in a diabetic cell culture model system

Experimental workflow: Primary retinal RMG were isolated from eyes of adult pigs. After 14 days in vitro, cells were treated in three different conditions for 72 hours. After treatments, cells were lysed and proteolysed proteins quantified by label-free LC-MS/MS-based proteomic analysis applying a data-independent acquisition mode.
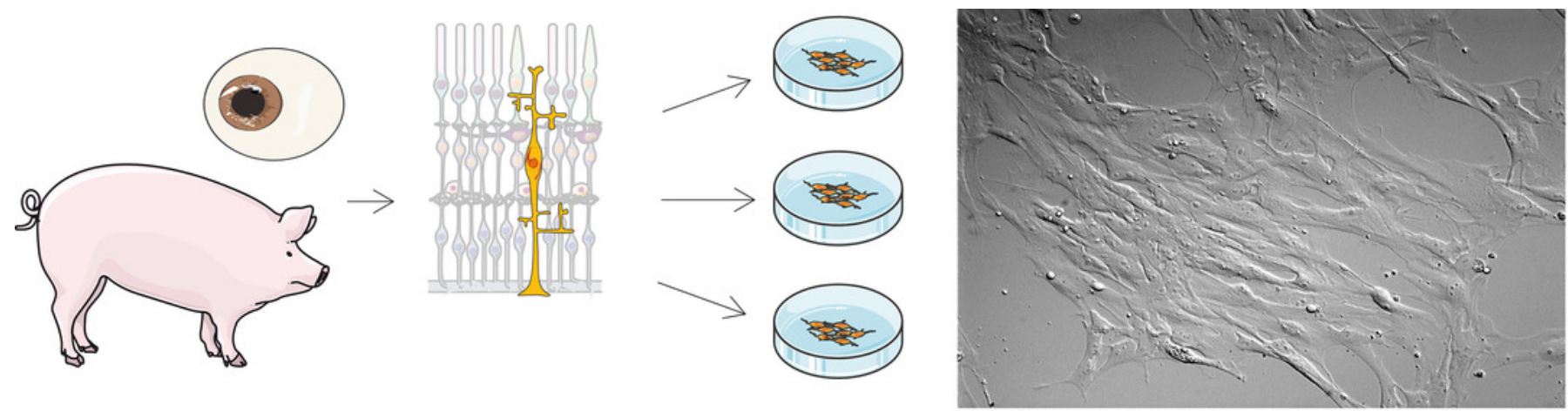

Isolation of primary porcine RMG

Culturing until day 14

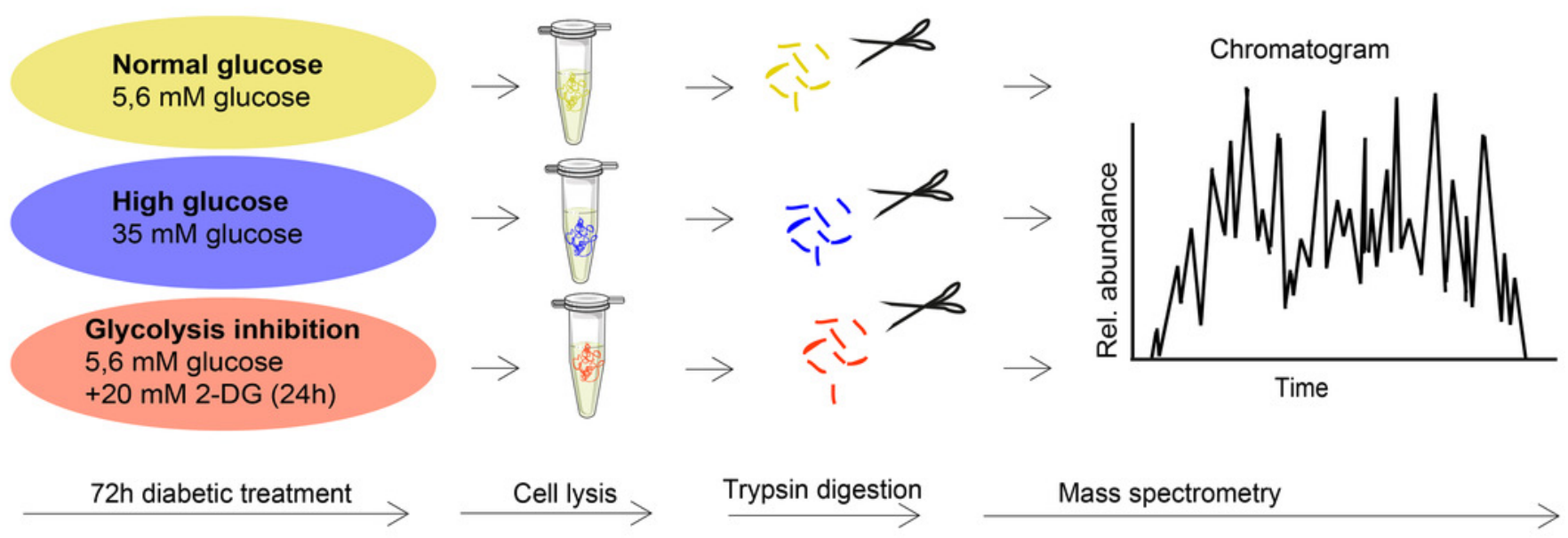




\section{Figure 2}

Differential protein abundances from primary porcine RMG in high glucose $(a, b)$ or glycolysis inhibition $(c, d)$ treatment compared to normoglycemic treatment group

Volcano plots illustrating the log2 transformed abundance fold change of quantified proteins (? 2 unique peptides) between the high glucose (a) or glycolysis inhibition (b) and normoglycemic group plotted against the corresponding negative log10 transformed $p$-value. Reference lines illustrate a $p$-value of 0.05 and a 1.33 -fold or 0.75 -fold differential abundance level. Significantly ( $p$-value $<0.05$ ) changed proteins with ? 1.33 (blue/red) or ? 0.75-fold (yellow) differential expression levels are colored. ECM-related proteins are labeled by gene names. (b), (d) Top ten most significantly altered biological processes in hyperglycemic (b) or glycolysis inhibition (d) treatment group according to Genomatix Gene Ranker Database. The three highest ranking processes in each condition belong to the context of ECM. 
a

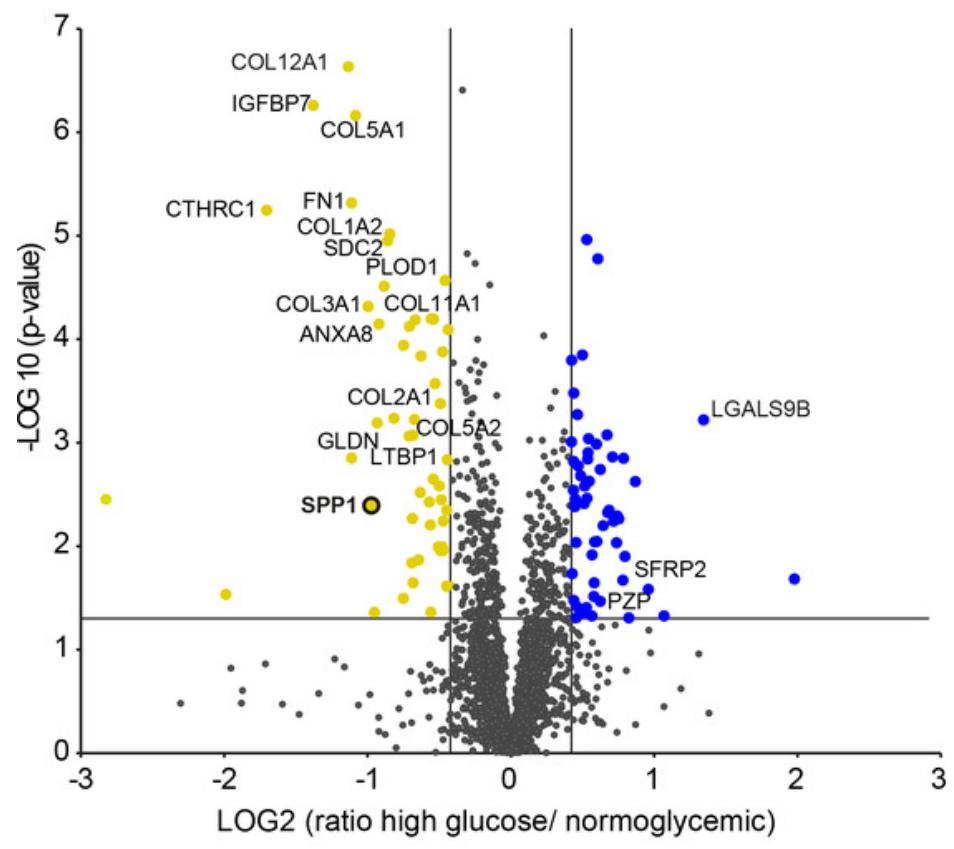

b

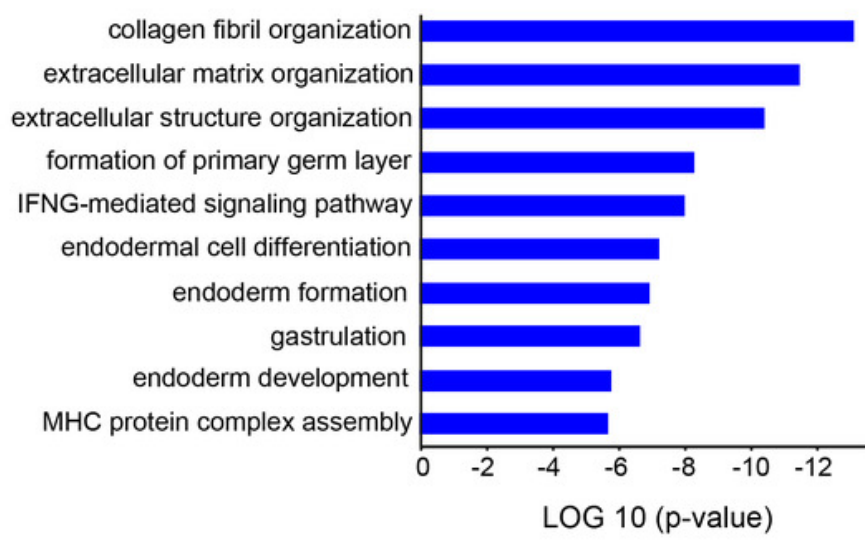

d

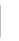

C

Glycolysis inhibtion vs. Normoglycemic

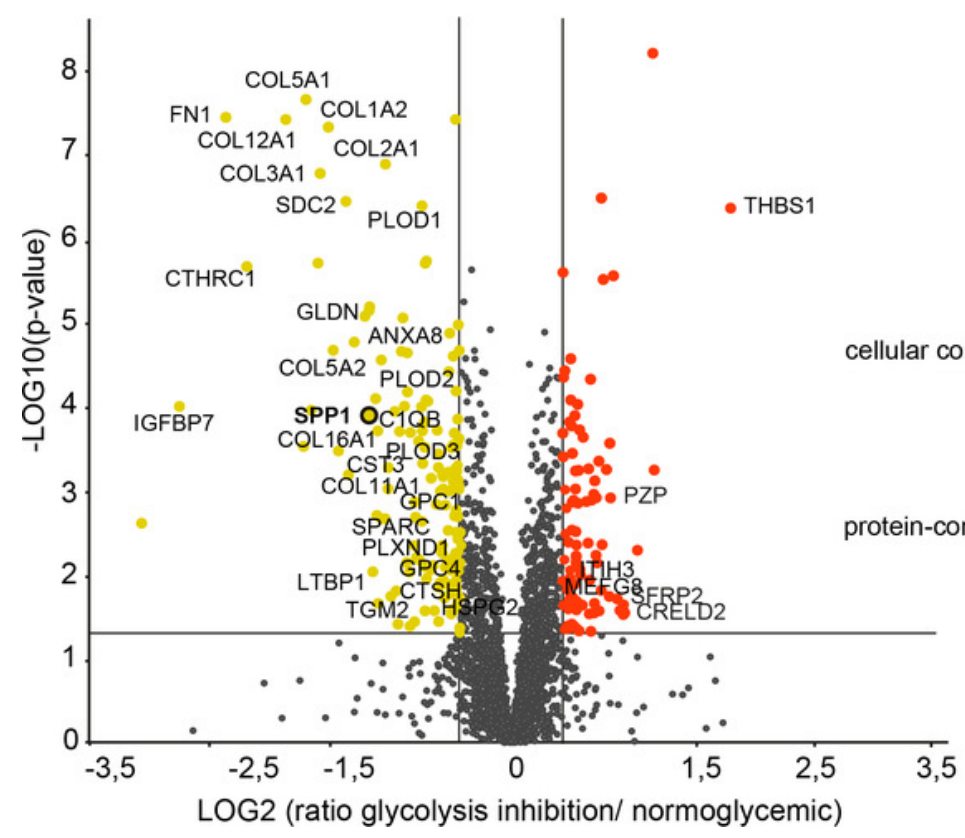

extracellular structure organization collagen fibril organization extracellular matrix organization ent organization and biogenesis cellular component organization response to endogenous stimulus complex subunit organizatio formation of primary germ layer response to stress

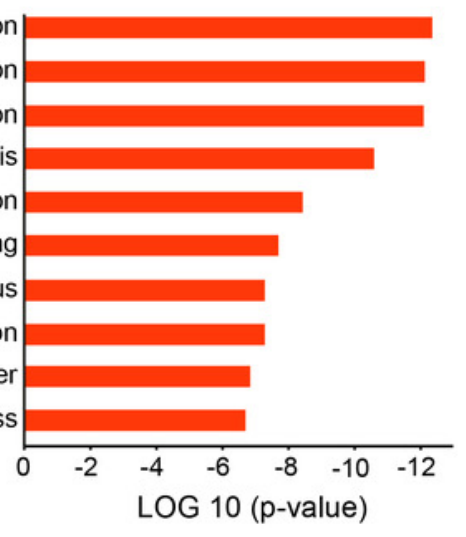




\section{Figure 3}

Overlap of proteins from primary porcine RMG with differential abundance in high glucose and glycolysis inhibition compared to normoglycemic treatment group points to affected ECM organization and cellular adhesions

Overlap of proteins with differential abundance in high glucose and glycolysis inhibition compared to normoglycemic treatment group points to affected ECM organization and cellular adhesions (a) Venn diagram of all proteins with differential abundance (criteria: $\geq 2$ unique peptides, p-value $<0.05$, fold change $\geq 1.33$ or $\leq 0.75$-fold) in hyperglycemic (blue) and glycolysis inhibition (red) compared to normoglycemic treatment group. (b) STRING protein functional association network of 65 overlapping proteins that display differential abundance in hyperglycemic and glycolysis inhibition compared to normoglycemic treatment group. Colored protein symbols belong to the GO term biological process "ECM organization" (yellow) and "cellular adhesion" (red).

a

Glycolysis inhibition

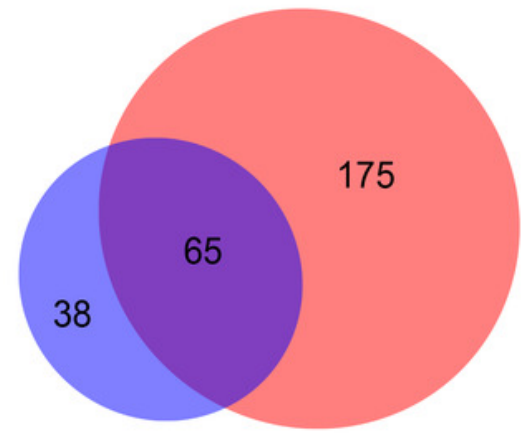

High glucose

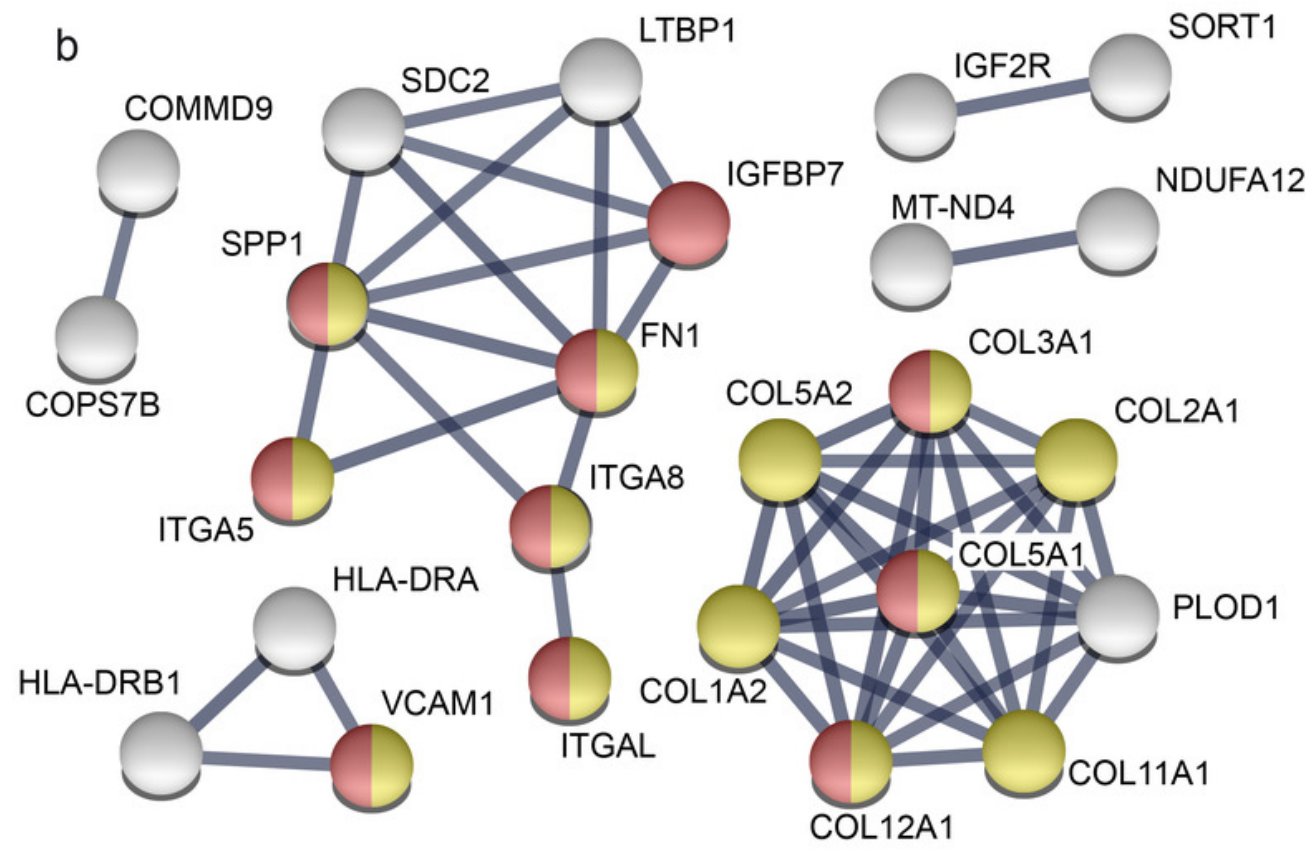

ECM organization
Cell adhesion 


\section{Figure 4}

Validation in a porcine diabetic retinal explant culture model

(a) Left: Nasal pole of a left pig eye, recognizable by the slight bulge of the eyeball (arrow). At the temporal pole, the corneal limbus tapers (asterisk). The optic nerve on the back of the eyeball is located slightly below the horizontal center and slightly temporal. Right: The same eye opened. A horizontal band extending from the nasal to the temporal pole nearby the optic disk (illustrated by $><$ ) is called "visual streak" and is distinguished by high densities of cones and ganglion cells (Garca et al. 2005; Hendrickson \& Hicks 2002). Explants were excised from this region dorso-nasal of the optic disc (circle). (b) Explants were cultured at the fluid/air interface on polycarbonate membrane with one drop of medium on the top. Treatments were performed for three days. 
a
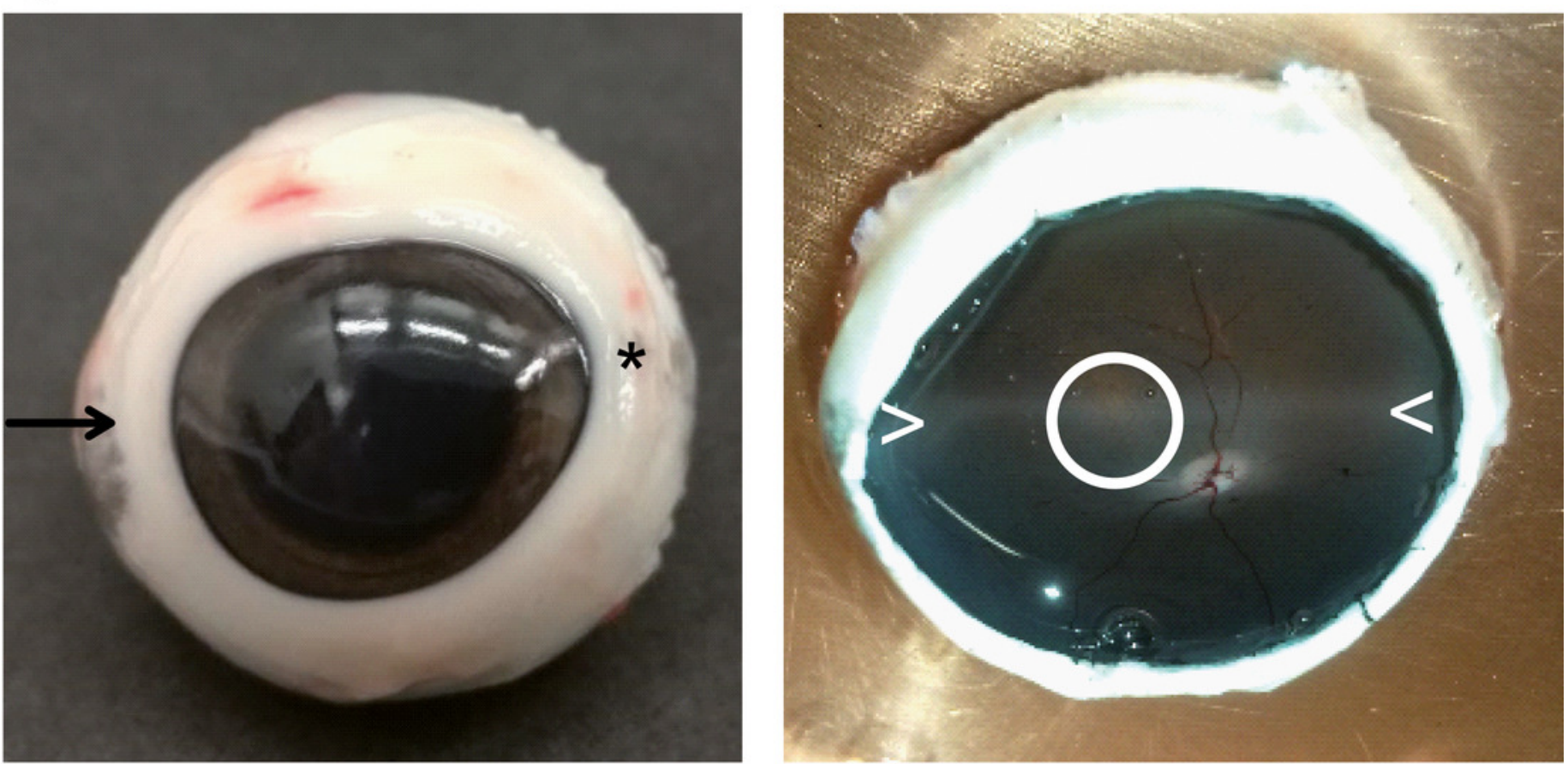

Selection and preparation of porcine retinal explants

b
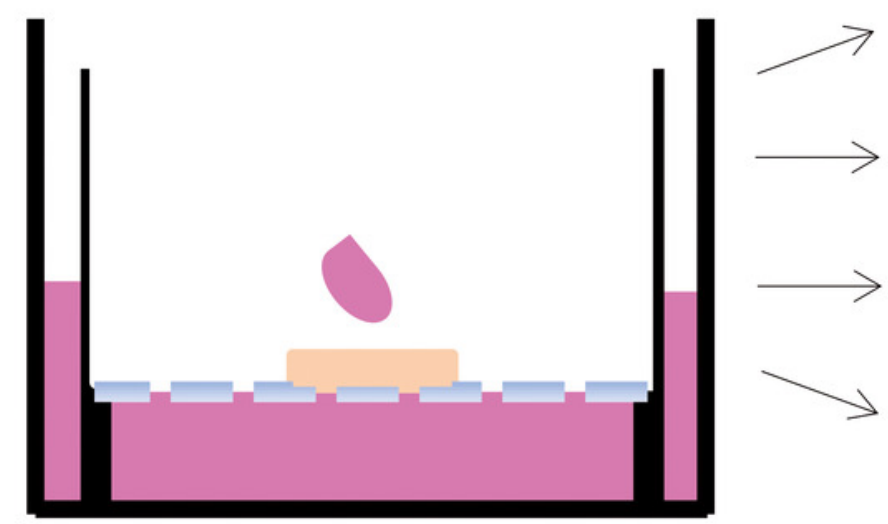

Normoglycemic $11 \mathrm{mM}$ glucose

\section{Osmotic control $11 \mathrm{mM}$ glucose} +59 mM mannose

\section{High glucose $70 \mathrm{mM}$ glucose}

Glycolysis inhibition $11 \mathrm{mM}$ glucose +40 mM 2-DG (24h)

\section{$72 \mathrm{~h}$ diabetic treatment}




\section{Figure 5}

Immunohistochemical verification of SPP1 abundances in retinal organotypic explants upon different treatment conditions

Retinal expression of SPP1 (left panel, green) and vimentin (mid panel, red) in representative explants cultured under normoglycemic $(n=4)$, osmotic control $(n=4)$, high glucose $(n=4)$ and glycolysis inhibition $(n=4)$ conditions for 72 hours. SPP1 was expressed at the level of POS independently of the culture condition. Expression along the inner neuroretina in the normoglycemic condition was associated with RMG, as demonstrated by the overlay image of SPP1 and vimentin stainings (top, right panel, yellow), but diminished in all other conditions. Nuclei are stained with HOECHST in blue. GCL, ganglion cell layer, NFL, nerve fiber layer, IPL, inner plexiform layer, INL, inner nuclear layer, OPL, outer plexiform layer, ONL, outer nuclear layer, POS, photoreceptor outer segments 


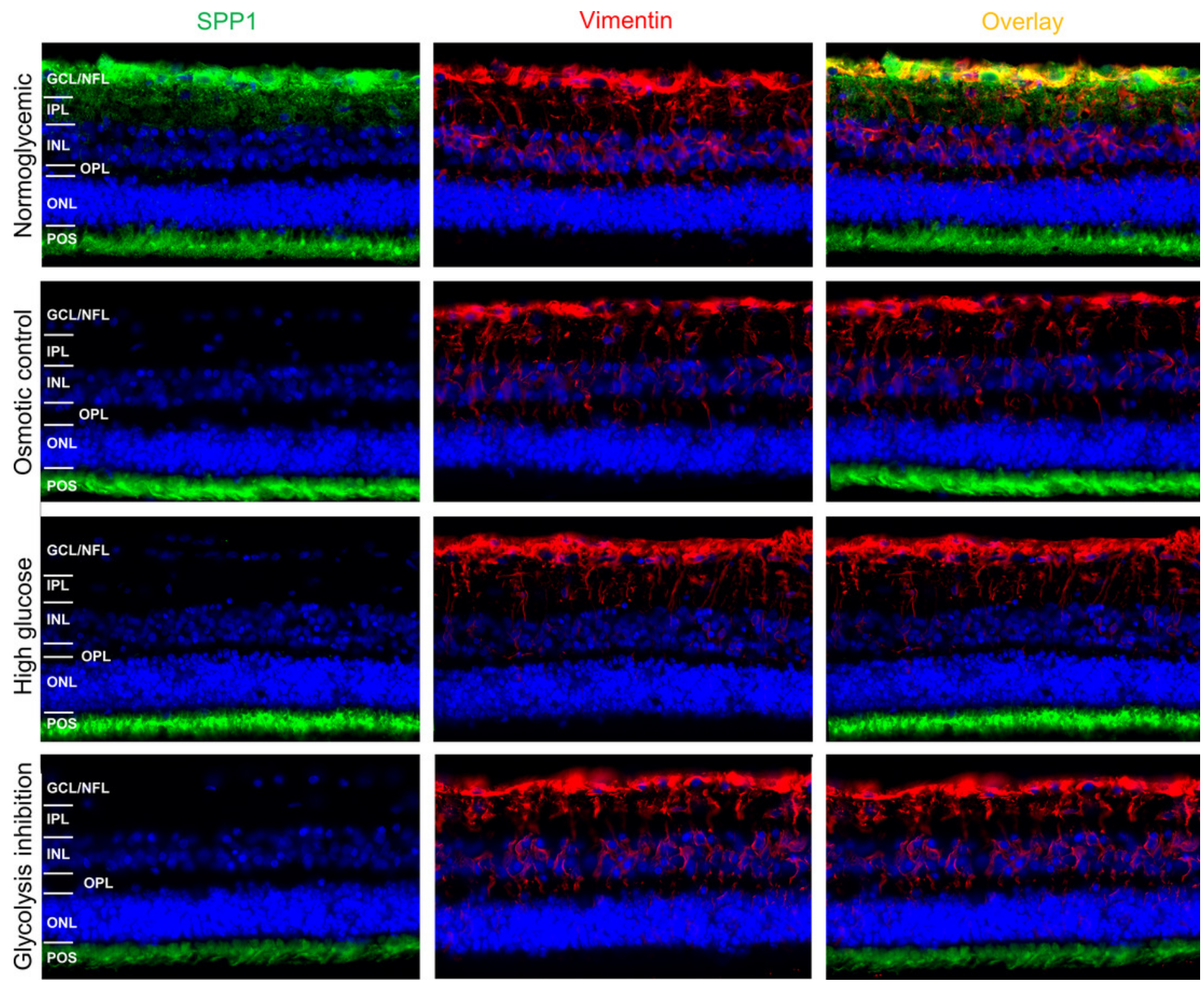

